\title{
InGaAs quantum disk: Fabrication via self-organization and spectroscopies
}

\author{
H KAMADA*, J TEMMYO*, E KURAMOCHI, H ANDO* and T TAMAMURA \\ NTT Basic Research Laboratories, 3-1 Morinosato Wakamiya, Atsugi, Kanagawa 243-01, Japan \\ *NTT Opto-electronics Laboratories, 3-1 Morinosato Wakamiya, Atsugi, Kanagawa 243-01, Japan
}

\begin{abstract}
We have examined the spontaneous rearrangement of a strained InGaAs/AlGaAs heterostructure, on a (311)B, substrate into naturally ordered array of InGaAs disks automatically buried in Al-rich alloy. Unlike nano-islands formed via Stranski-Krastanov mechanism, it serves as a strong tendency to align themselves. We have stressed important interplay of islanding of the material with lattice mismatch, atomic diffusion across the interface between unstrained and strained materials, lateral mass transport, and development of the surface into low index surfaces with low surface energies: all these seek to lower the total energy. Because of the damageless fabrication, these quantum disks showed excellent optical properties, which facilitated single-dot spectroscopy. Such spectroscopy revealed that lateral together with vertical confinement of exciton motion discretizes the exciton density-of-states resulting in sharp and distinct photo emission/absorption spectra despite their mesoscopic confinement. These characterize optical properties which are specific only to zero-dimensional system, thereby proving quantum dot characteristic. As for future device application, we undertook an attempt to artificially position the self-organized structure more accurately by periodic seeding on the virgin (311)B substrate. The results show that it is feasible to further improve the ordering of the array of quantum disks.
\end{abstract}

Keywords. InGaAs quantum disk; self-organization; spectroscopies.

\section{Introduction}

The semiconductor zero-dimensional (0D) structure has long been a subject of interest not only in physics but also in device application. The effort to realize the semiconductor OD structure is now coming by a new apex following the application of the so-called selforganized dot formation (Leonard et al 1993, 1994; Mazin et al 1994). The spontaneous rearrangement of a strained InGaAs/AlGaAs heterostructure on a (311)Bsubstrate into naturally ordered array of InGaAs disks, which are automatically buried in Al-rich alloy, is one such phenomena (Nötzel et al 1994a, b). It has been shown that extremely dynamic atomic rearrangement of indium and aluminum in the InGaAs/AlGaAs hetrostructure enables energy lowering through the island formation that relaxes the strain, atomic diffusion across the interface and rearrangement of the surface into low index surfaces, all of which are driven essentially by the strain within the material. The extent of the resulting disk-shaped structure is as low as $30-100 \mathrm{~nm}$ in diameter, and it is controlled by the strain, i.e. by the composition of InGaAs (Nötzel et al 1995b). As the lateral extent of the structure is a few exciton Bohr radii, we may observe the discrete energy level structure of exciton (Notomi

\footnotetext{
*Author for correspondence
}

et al 1996; Kamada et al 1997, 1998). An electron and a hole in a disk interact much more strongly in a $0 D$ system than in a two-dimensional system; both exciton effect as well as the exciton-exciton correlation may be greatly enhanced (Brunner et al 1994; Forchel et al 1996; Kamada et al 1998).

We shall review the result of structural analysis followed by the result of spectroscopical study. Low temperature microscope photoluminescence spectroscopy has revealed a number of sharp distinct single-dot exciton luminescence lines (the narrowest FWHM $<34 \mathrm{meV}$ ), and their discrete excited state resonances (Notomi et al 1996; Kamada et al 1997, 1998). Each dot emits not only the exciton but also biexciton luminescence (Kamada et al 1998). The biexciton effect plays an important role in optical nonlinear response of the quantum dot system. Finally, we shall introduce our recent attempts to position the self-organized structure artificially and more accurately by adding periodic artificial seeding on the virgin (311)B substrate (Kuramochi et al 1997).

\section{Fabrication of quantum dot via self-organization}

We have examined a disk-shaped nanostructure grown via an unique spontaneous reorganization of InGaAs/ AlGaAs heterostructure grown on a (311)B substrate. This spontaneous reorganization results in arrays of well- 
ordered InGaAs disk-shaped structures, with diameter of 30-100 nm, automatically embedded in AlGaAs (Nötzel et al 1994a, b). Detailed growth conditions have been reported previously (Nötzel et al 1994a, 1996). In brief, the samples were all grown by low-pressure metalorganic vapour phase epitaxy on GaAs (311)B-substrates, using trimethyl-gallium (TMG), -aluminum (TMA), -indium (TMI), and arsine as gas sources. Typically, following the deposition of a $100 \mathrm{~nm} \mathrm{Al} \mathrm{Al}_{0.5} \mathrm{Ga}_{0.5} \mathrm{As}$ buffer layer, an InGaAs layer with the nominal thickness of $15 \mathrm{~nm}$ as an initiating layer, a $150 \mathrm{~nm}$ thick $\mathrm{Al}_{0.5} \mathrm{Ga}_{0.5} \mathrm{As}$, and il thinner InGaAs disk layer of 3-6 nm in nominal thickness were grown successively. This was followed by 1-3 min growth interruption for spontaneous disk formation (Nötzel et al 1994b, 1996). The growth temperature was between $690^{\circ} \mathrm{C}$ and $800^{\circ} \mathrm{C}$. During the growth the initiating layer, already formed itself into threedimensional structure with thickness modulation. It is the last thinner InGaAs layer that converts during the growth interruption into arrays of thin InGaAs disks which are automatically covered with aluminum-rich alloy, and there is development of the nanocrystal surface into low index surface.

Resulting structure can be easily studied for the larger nanocrystals formed for smaller indium composition. In figure 1, we present surface topography obtained by secondary electron microscopy (SEM) of a sample grown nominally with $\mathrm{In}_{0,2} \mathrm{Ga}_{0.8}$ As of $10 \mathrm{~nm}$ thickness. It was grown at $800^{\circ} \mathrm{C}$. The SEM image shows well-ordered arrays of nanocrystals. The direction of alignment is about $45^{\circ}$ off the [011] azimuth and is not along any specific step edge direction of the initial (311)B surface. The top view (upper figure) suggests that the positional correlation is connected with the faceted surface of the nanocrystals rather than with preferential nucleation associated with the step structure of the initial surface. The evolution of the crystal facets during the early stage of the formation of the nanocrystal together with the break up of the InGaAs layer selects distinct directions for surface migration or strain relaxation between the nanocrystals that can result in the observed ordering. The cross-section of the structure is also shown in figure 1 (lower) after stain etching. It demonstrates automatic coverage on the InGaAs disk by aluminum-rich alloy. This inevitably indicates that during the selforganization a very large number of aluminum atoms in the underlying AlGaAs moves toward surface and finally penetrates the InGaAs layer. Scenario of the self-organization is schematically represented in figure 2 and is summarized as follows: At an early beginning, following the deposition of InGaAs, islanding to reduce strain energy of the system is initiated, which results in wavy surface. Because of the high growth temperature, counter-diffusion of indium and aluminum atoms across the initial InGaAs/AIGaAs interface occurs, resulting in
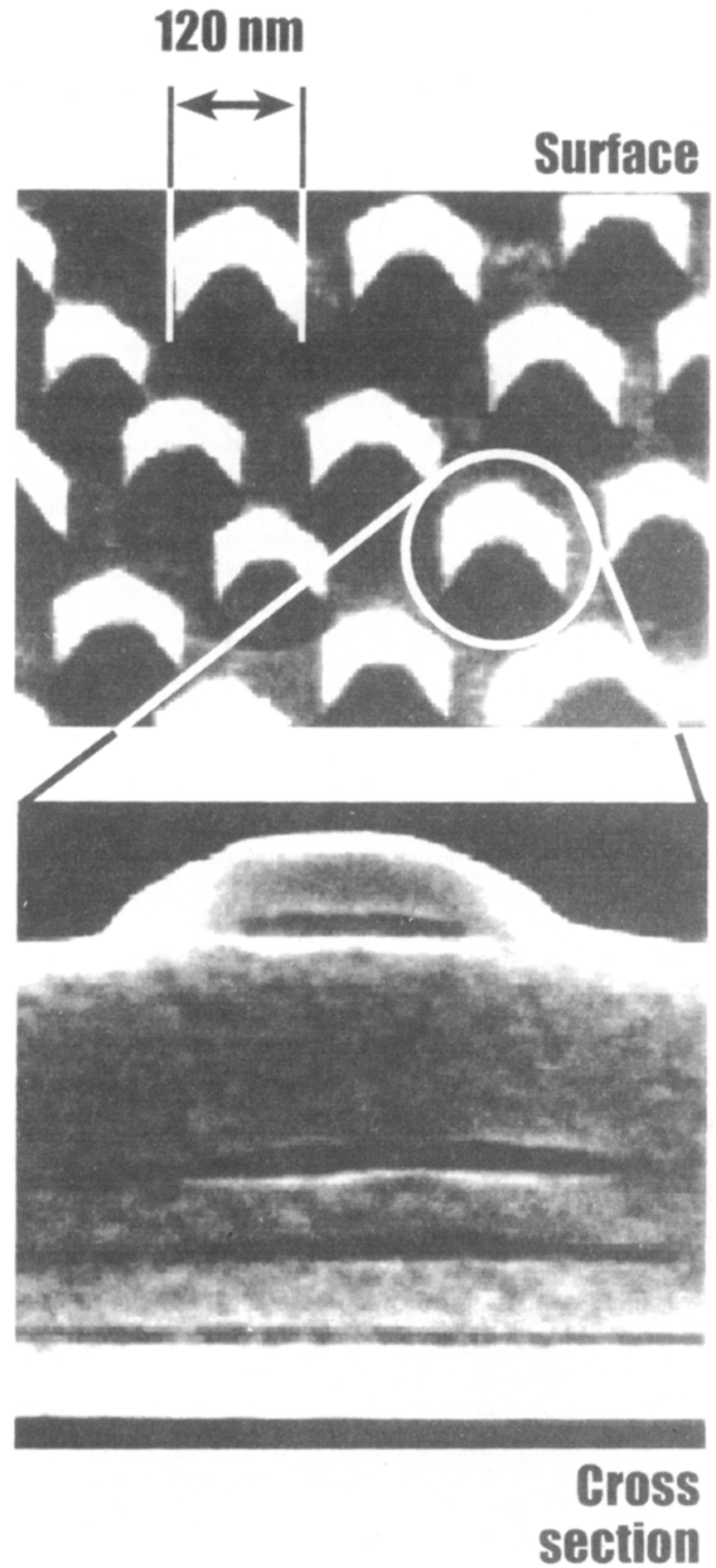

Figure 1. Secondary electron microscopy images (SEM) of surface nanocrystals spontaneously formed from $10 \mathrm{~nm}$ $\mathrm{In}_{0.2} \mathrm{Ga}_{0.8} \mathrm{As} / \mathrm{Al}_{0.5} \mathrm{Ga}_{0.5} \mathrm{As}$ on (311)B face. Upper figure is the top view and lower figure shows the cleaved (110) facet after stain etching, revealing embedded structure. 
alloying and thereby the total strain energy is again lowered by reduction of lattice constant to that closer to GaAs. Subsequent mass transport of aluminum-rich alloy covers up the InGaAs islands and it may further reduce the total energy leaving the surface of strained islands covered by less-strained material. Simultaneously, the surface of each nanocrystal develops itself from the initial (311)B of a high surface energy into low-index surfaces such as (100), (110) and (111), all of which have low surface energies. As a result, there remains no wetting layer which is commonly observed in the systems formed via Stranski-Krastanov growth mode switching mechanism (Leonard et al 1993, 1994).

The growth mechanism is therefore understood to be governed by surface energy, strain and dynamic surface migration. Generally, strained layers are unstable against the formation of wavy surface and islanding which reduce the total energy. Such tendency is enhanced on high-index surface due to the initial high surface energy. It may also increase the surface roughening. The high density surface step distribution may then facilitate the atomic mixing through the interface at high growth temperature by frequent exchange of the atomic sites between both edges of the steps at the interface. Thus the atomic counter-diffusion between AlGaAs and InGaAs allows the lowering of the total energy to form an unstrained (or less strained) surface by this alloy mixing, resulting in coverage by aluminum-rich alloy from the buffer layer.

The decreasing size of nanocrystals and InGaAs disks for increasing indium composition is consistent with the smaller island size at higher strain energy. The uniformity in size and shape of the nanocrystals is however maintained with size reduction (Nötzel et al 1995). This is shown in the histograms in figure 3 of the base length and the height of the large and small microcrystals, one with $x_{\mathrm{ln}}=0.2$ (figure $3 \mathrm{a}$ ) and $x_{\mathrm{In}}=0.4$ (figure $3 \mathrm{~b}$ ). For both samples, the size distribution of the base length and the height is well within $10 \%$ of the average values. The shape of the nanocrystals, characterized by the ratio of the base length to the height, does not change with the size.

The mean distance of the nanocrystals is directly related to the nominal thickness of the InGaAs layer. This behaviour is shown in figure 4 for $\mathrm{In}_{0.4} \mathrm{Ga}_{0.6} \mathrm{As}$ layer. While the average distance decreases with the increase of the InGaAs layer thickness, the base length and the height remain almost constant. On the other hand, for the same InGaAs layer thickness, the average distance does not depend on the indium composition, i.e. on the size. Therefore, we can independently control the size and distance by the indium composition and the thickness.

The self-organization of InGaAs/AlGaAs on (311)B face is driven by a nonlinear cooperative and competitive phenomenon. Deposition of highly strained material creates an instability which is resolved by cooperative rearrangement of materials. Ordered structure is in essence an inequilibrium against long-term stability; we find remarkable resemblance to the natural patterns such as the coat marking of cheetahs (Temmyo and Tamamura 1997; Temmyo et al 1997): fingerprint of life as an inequilibrium state of nature. (a)
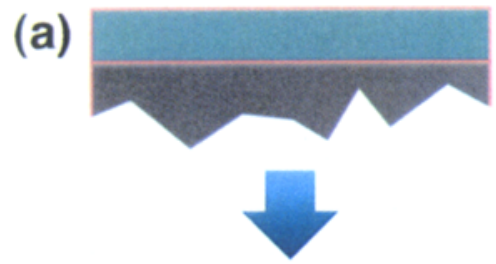

(b)

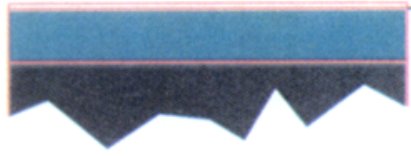

(e)

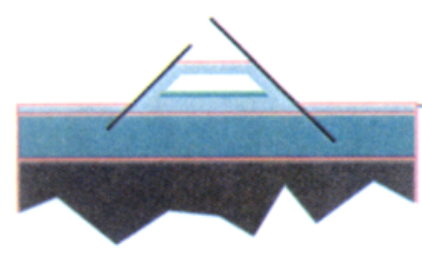

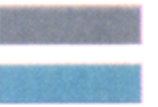

GaAs

AlGaAs

InGaAs

InAIGaAs

Initial interface
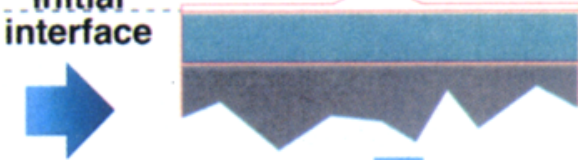

(c)

Initial
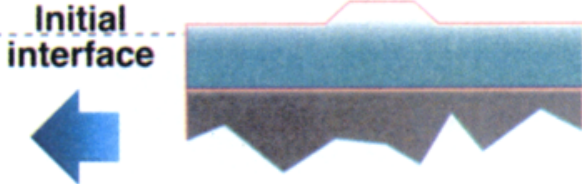

(d)

Figure 2. Schematic representation of a sequence of self-organization on (311)B face. Initial stage of InGaAs deposition (a), developing InGaAs islanding (b) to (c), alloy mixing across InGaAs/AlGaAs interface (d), coverage by AlInGaAs alloy via lateral mass transport, and development of nanocrystal surface into low index surfaces (e). 


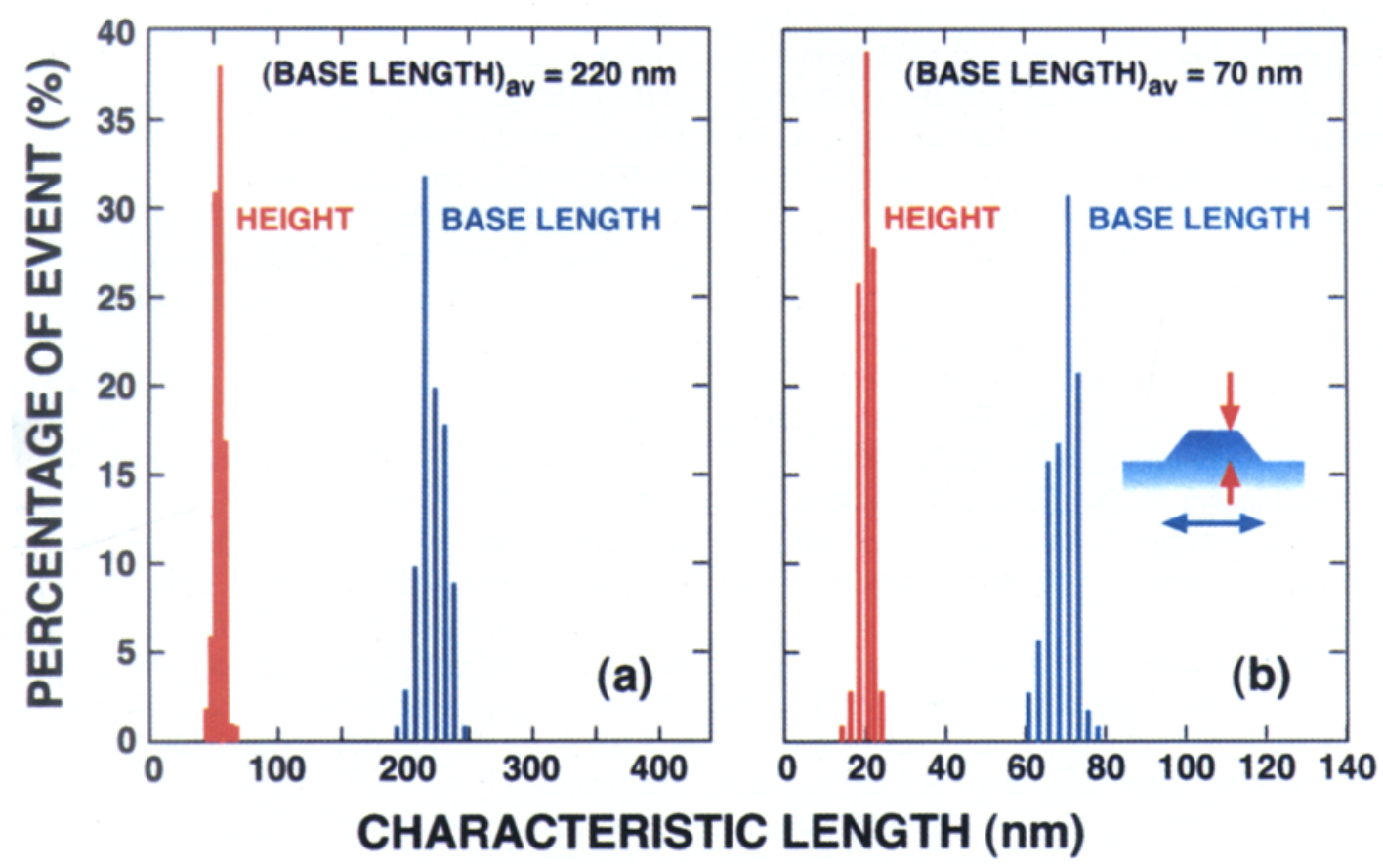

Figure 3. Histogram of nanocrystal geometry: Base length measured at half height and of height of nanocrystais are plotted for two samples. (a). with $220 \mathrm{~nm}$, and (b). with $70 \mathrm{~nm}$ average base length. They correspond respectively to indium composition of 0.2 and 0.4 .

\section{Single-dot spectroscopy}

\subsection{OD exciton luminescence emission}

In the quantum disk, the strongest confinement is along the growth direction like in quantum well. The full quantization of the energy spectrum of a $2 \mathrm{D}$ system into a zero-dimensional (OD) structure by an additional lateral confinement makes totally discritized level structure like in atoms. Physical properties should therefore reflect such density-of-states spectrum which are specific only to OD structure. Spectroscopic evidences of discrete nature of electronic level structure in OD semiconductor nanostructures is obviously an ultimate proof of quantum confinement effects. While optical detection and spectroscopy of single absorbers in variety of media are ultimate experimental ways to examine the interplay of various interactions leading to the relaxation of the optical dipole moment, in atomic and molecular physics, a number of spectroscopic methods have been developed. Common obstacle towards such spectroscopies is broadening of the spectrum due to the statistical distribution of absorbers. Correspondingly, in variety of semiconductor dot systems, ever reported, distribution of dots in size and composition is the primary cause of the broadening. What is essentially in need is thus a single-dot spectroscopy where a small number of individual dot luminescences are to be observed of spectral linewidth much smaller than distribution width.

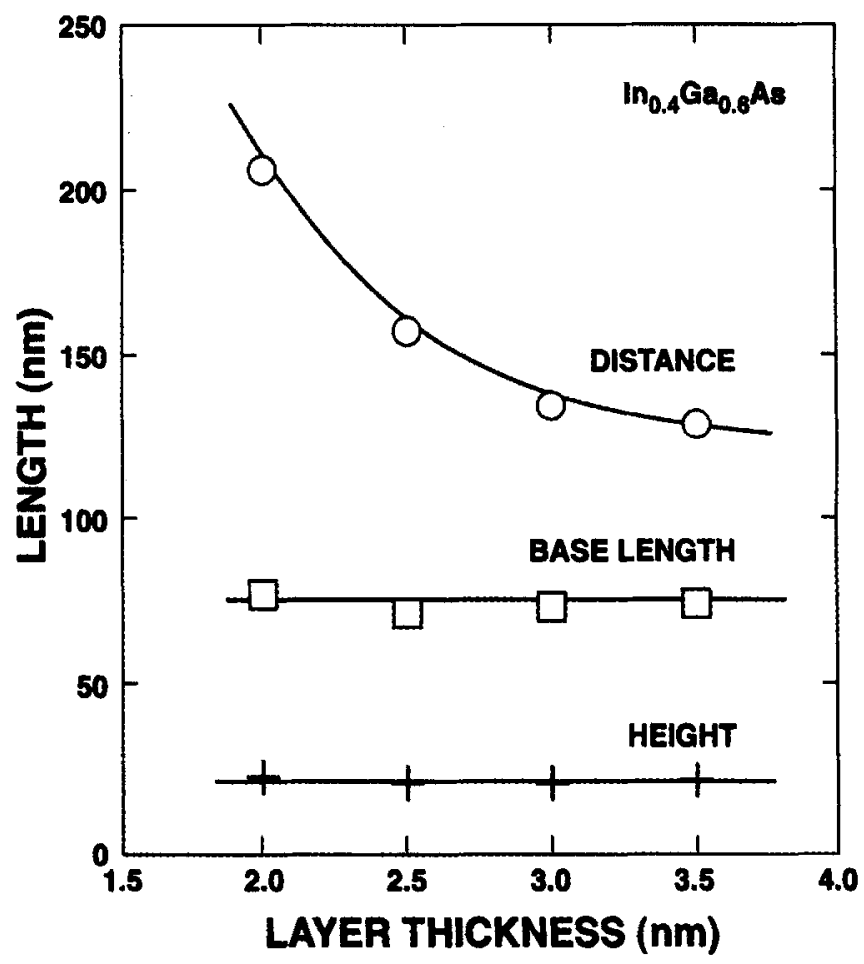

Figure 4. Dependence of average distance (square root of area density), base length, and height of nanocrystals on $\mathrm{In}_{0.4} \mathrm{Ga}_{1.6} \mathrm{As}$ layer thickness. 
According to the standard prescriptions toward singledot spectroscopy, we have undertaken microscopic photoluminescence spectroscopy (Grundmann et al 1995; Notomi et al 1996). We selected few dots and observed distinctive narrow luminescences by selecting them using their unique individual optical properties (Notomi et al 1996; Kamada et al 1997). Also, to further reduce the number of dots within a spot and to facilitate repeated spectroscopy on specific areas, a metal mask with fine openings (down to $0.2 \mu \mathrm{m}^{2}$ ) was processed on the sample surface coated with a thin dielectric film ( $200 \mathrm{~nm}$ thick). A laser beam from a continuous-wave laser was then focussed to a $2 \mu \mathrm{m}$ spot on the sample surface and the luminescence was collected through the same lens.

A number of sharp (typical FWHM less than $0.2 \mathrm{meV}$ and the narrowest less than $30 \mu \mathrm{eV}$ ) luminescence lines with very high radiative efficiency were observed. Typical low-temperature micro-PL spectra taken under an excitation of $514.5 \mathrm{~nm}$ line of an $\mathrm{Ar}^{+}$ion laser are shown in figure 5 for a sample of $3 \mathrm{~nm} \mathrm{In}_{0.35} \mathrm{Ga}_{10.65}$ As (Kamada et al 1996). In the same sample, the narrowest lumi- nescence line ever observed is only as low as $34 \mu \mathrm{eV}$ (after correction of spectrometer resolution it values well below $30 \mu \mathrm{eV}$ ) (Kamada et al 1997). Such a small linewidth in turn reads a dephasing time of more than 40-50 ps; implying suppression of scattering events at luminescent state, which is essentially due to the discrete density-of-states. While under very low excitation only a few very narrow lines are visible, under increasing excitation intensity new lines emerge and grow with excitation. All lines appear, however, to reach saturation intensity even while under a low pumping intensity; e.g. a PL line near the centre of distribution reaches saturation even under an excitation of $54.9 \mathrm{nW}$ per spot of $2 \mu \mathrm{m}$ in diameter. At highest excitation, these lines overlap due to the substantial broadening under such strong pumping condition and they appear to converge into a broad line of Gaussian distribution (figure $5 \mathrm{c}$ ). Indeed the overall spectrum coincides with that taken under the conventional PL of macroscopic probe size (Marzin et al 1994; Fafard et al 1995; Grundmann et al 1995; Gammon et al 1996; Kamada et al 1997).

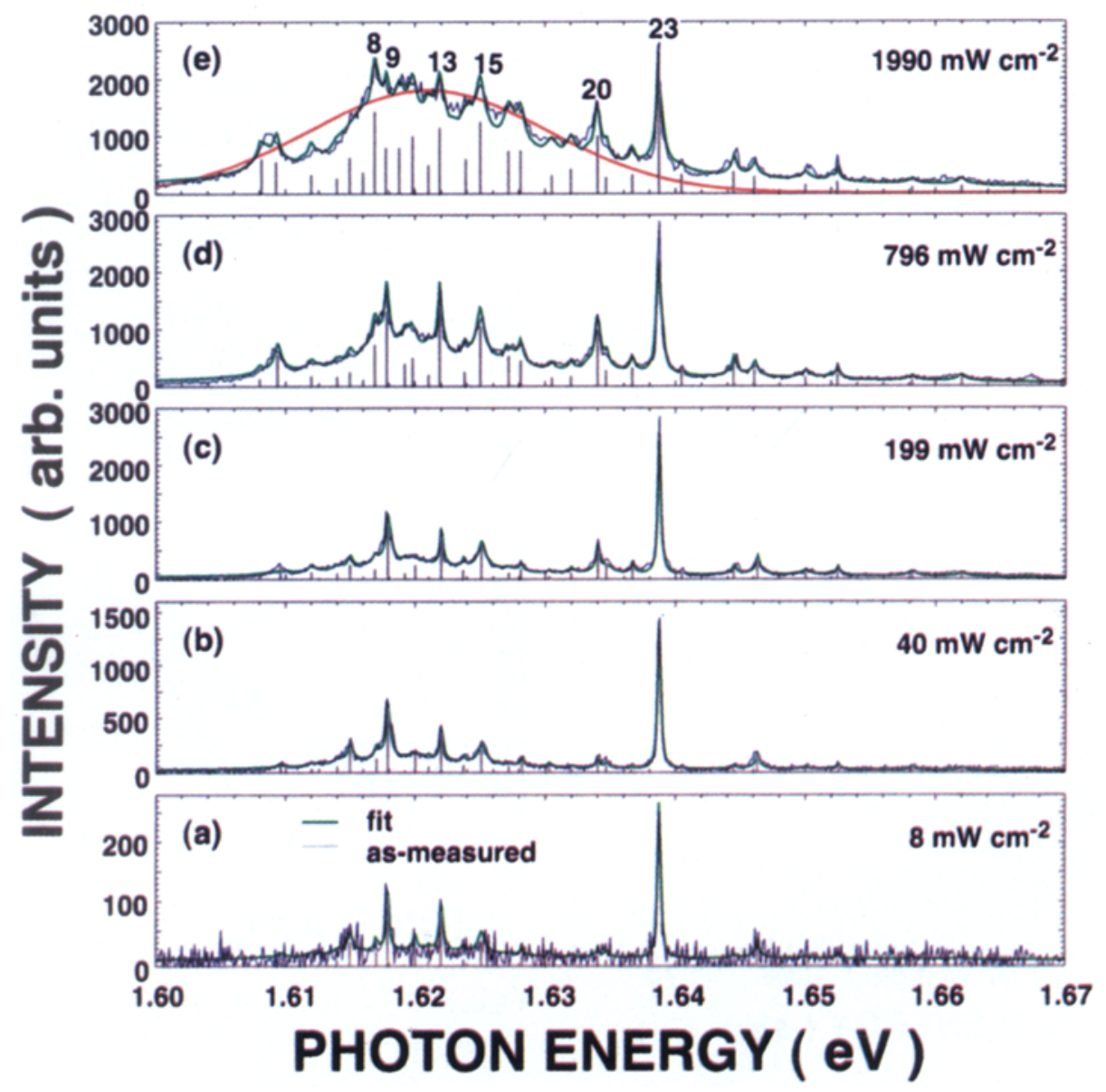

Figure 5. Evolution of microscope dol luminescence spectrum at $10 \mathrm{~K}$ in a $3 \mathrm{~nm}$ $\mathrm{In}_{0,3} \mathrm{Ga}_{9.7} \mathrm{As}$ sample taken under a series of excitation intensity. Also shown with each experimental spectra are lineshape fitting either with Lorentzian or that with tail broadening. Excitation by $514.5 \mathrm{~nm}$ line of $\mathrm{Ar}^{+}$ion laser was used. 
Figure 6 shows a similar series of micro-PL spectra recorded under excitation of different energies. The sample used here is composed of a $3 \mathrm{~nm} \mathrm{In}_{0,3} \mathrm{Ga}_{0,7} \mathrm{As}$ layer left for spontaneous reorganization during growth interruption. As in figure 5, series of sharp luminescence lines are resolved, whose number and intensities are clearly dependent on the excitation energy: when the excitation energy exceeds the $\mathrm{Al}_{0.5} \mathrm{Ga}_{0.5} \mathrm{As}$ direct gap, a markedly large number of intense lines emerge, while for lower-energy the excitation selects only a fraction of lines. Notice also that when $E_{\text {laser }}$ is around $1.8 \mathrm{eV}$, a bunch of PL lines near $1.68-1.70 \mathrm{eV}$ are enhanced, showing that the excitation is resonant with excited states of dots in that energy range. When the excitation is resonant within the $\mathrm{Al}_{0.5} \mathrm{Ga}_{0.5} \mathrm{As}$ (barrier) band, photocreated carriers can feed all the low-lying dots, and thereby all the dots in the spot may emit luminescence. In contrast, under an excitation below barrier energy gap, only a small fraction of dots, whose excited states are resonant by chance are excited, since the carrier transfer between the different dots is presumably absent (Kamada et al 1997). It is very interesting to note that when the quantum dot is excited resonantly with a discrete excited state resonance, the saturation and line broadening of luminescence does not occur at the same excitation density which we could observe under abovebarrier excitation. Under resonant excitation condition, it required an excitation density which is higher by nearly three orders of magnitude than under above-barrier

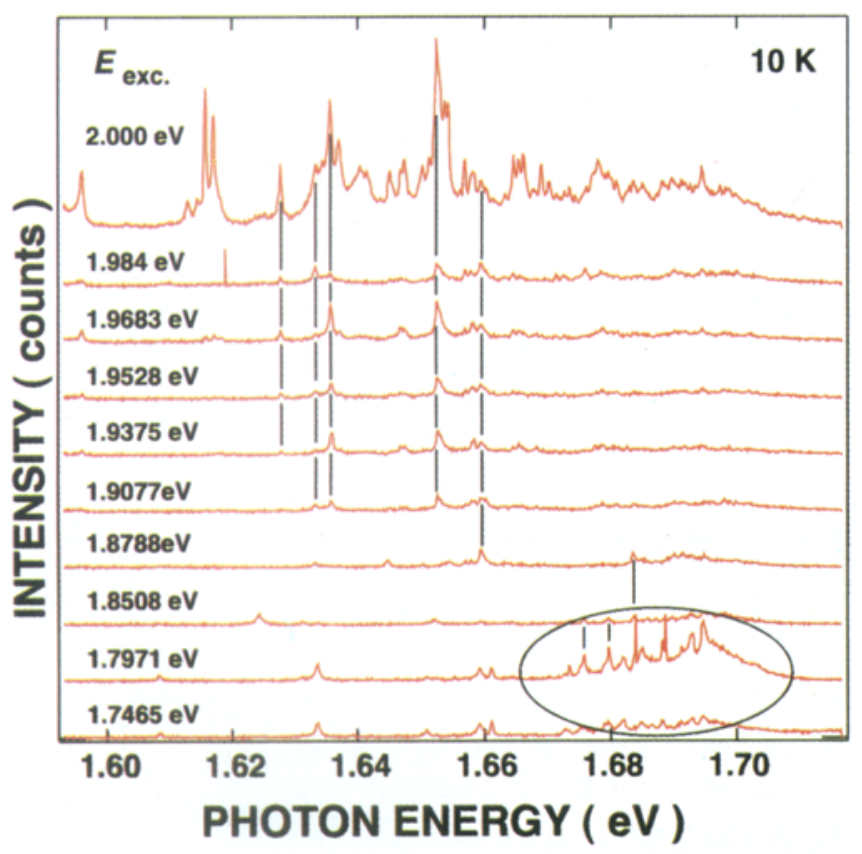

Figure 6. Microscope $\mathrm{PL}$ spectra taken on a $3.5 \mathrm{~nm}$ $\mathrm{In}_{0.35} \mathrm{Ga}_{0.65}$ As sample with various excitation energies at $10 \mathrm{~K}$. Barrier material is $\mathrm{Al}_{0.5} \mathrm{Ga}_{0.5} \mathrm{As}$. Some $\mathrm{PL}$ lines indicated by vertical bars or enclosed are enhanced in the oval resonantly under below-barrier excitation. excitation. This is due to Pauli exclusion principle. While the dot is under the above-barrier excitation, more than two excitons can be easily fed from the barrier into the dot and thereby state filling can be reached even at very low excitation. Essential cause of such behaviour is again the discrete density-of-states of the quantum dot (disk).
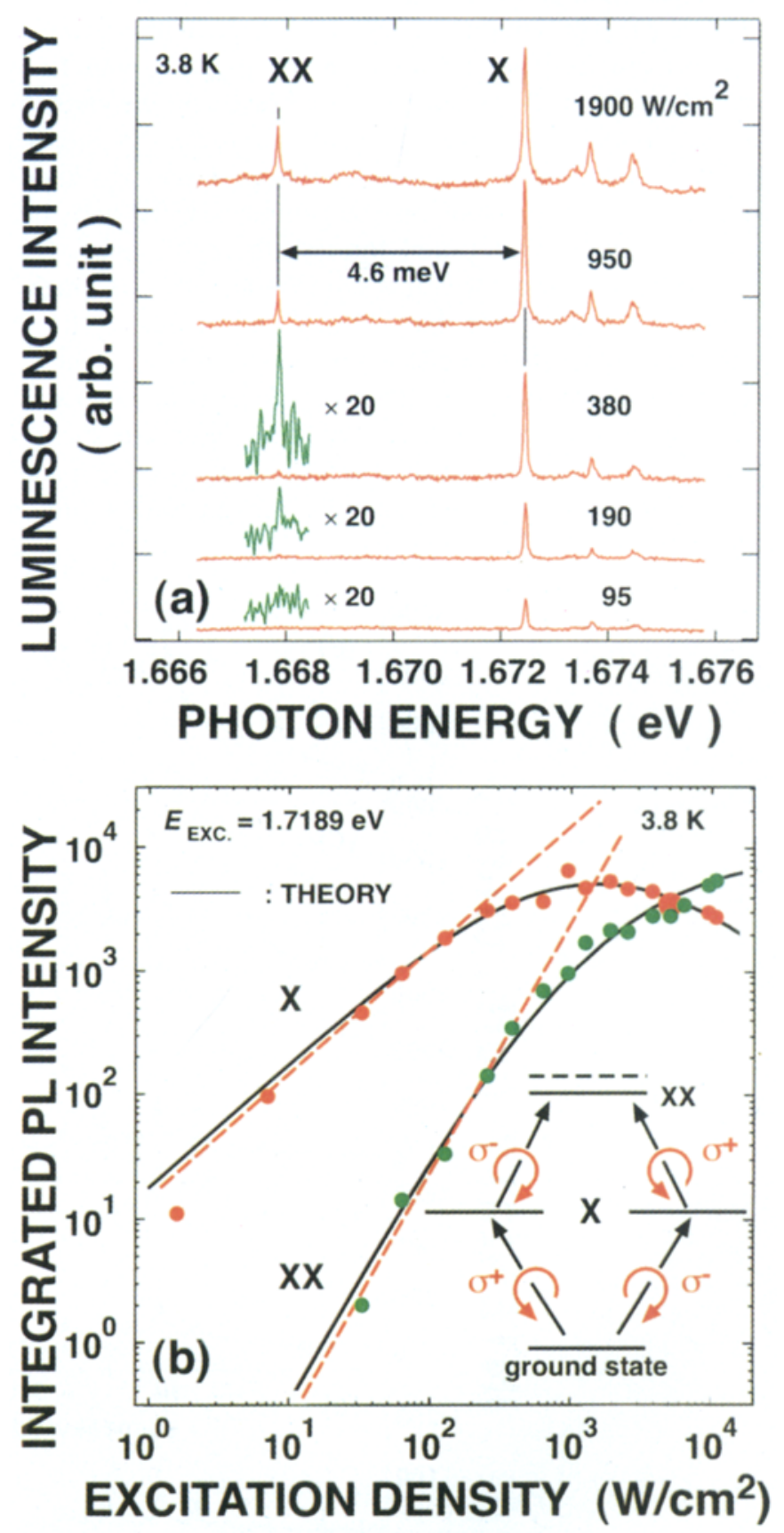

Figure 7. (a) Evolution of series of luminescence lines under increasing excitation density $\left(95-1900 \mathrm{~W} / \mathrm{cm}^{2}\right)$. Excitation energy is $1.7819 \mathrm{eV}$. Exciton and biexciton luminescence are labeled $\mathrm{X}$ and $\mathrm{XX}$, respectively. (b) Integrated luminescence intensity of exciton and biexciton in a single InGaAs quantum disk at $3.8 \mathrm{~K}$ plotted as a function of excitation density. Inset shows exciton-biexciton four-level scheme with hericity-dependent transitions. 


\subsection{Biexciton population}

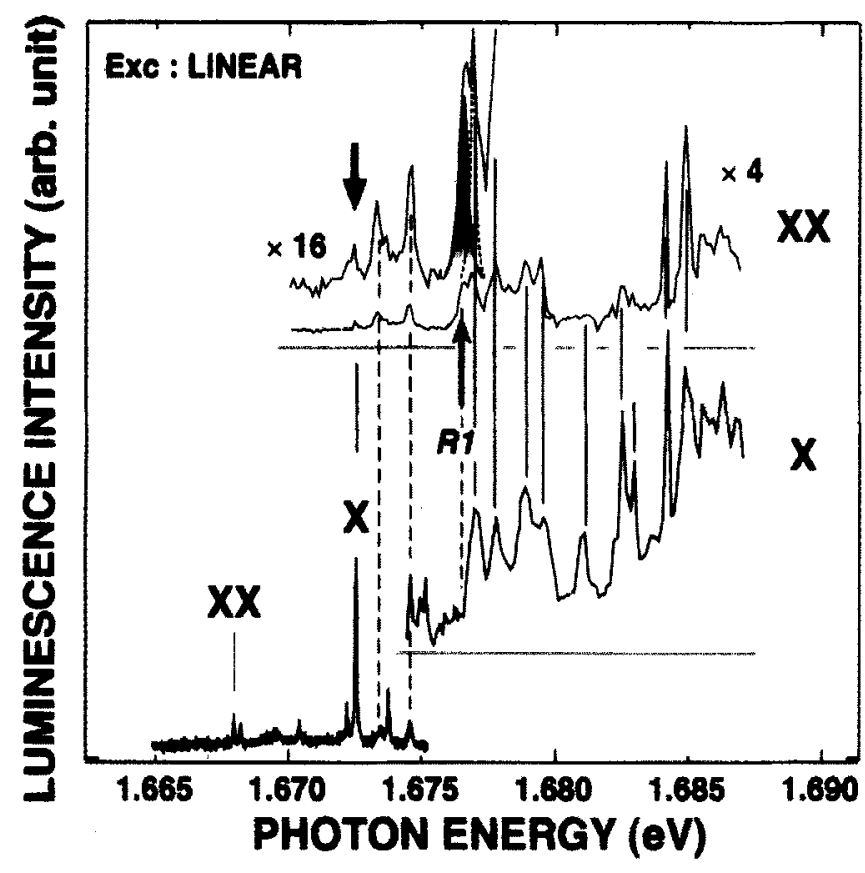

Figure 8. Photoluminescence excitation spectra taken on exciton (X) and biexciton (XX) lines at $4 \mathrm{~K}$ under an excitation density $\left(\sim 100 \mathrm{~W} / \mathrm{cm}^{2}\right)$. Excitation source is linearly polarized. Vertical bars indicate correspondence between resonances in $\mathrm{X}$ and $\mathrm{XX}$. The line labeled $R I$ appears only in the XX-PLE spectrum.
While looking into a few number of dot luminescences at a time, we found another important feature of the OD system. Figure 7a shows a typical spectrum involving only a few dot luminescence lines taken through a $0.2 \mu \mathrm{m}^{2}$ pin hole. Sample was composed of nominally a $3 \mathrm{~nm}$ thick $\mathrm{In}_{0.4} \mathrm{Ga}_{0.6} \mathrm{As}$, and the estimated disk lateral extent was $30-50 \mathrm{~nm}$. Excitation was linearly polarized. It demonstrated biexciton population in a single dot: with increasing excitation density, the exciton line labelled $\mathrm{X}$ of a linewidth less than $80 \mu \mathrm{eV}$ (spectrometer resolution of $30 \mu \mathrm{eV}$ ) grows linearly, saturates to a maximum and then falls into fading. Another distinct sharp line of the biexciton transition labeled $\mathrm{XX}$ at an energy lower by $4.7 \mathrm{meV}$ first emerges for increased excitation density and increases quadratically. It continues growing even after the exciton line saturation. Such intensity evolutions are summarized as figure $7 \mathrm{~b}$, where the integrated PL intensity as a function of excitation density is plotted for $X$ and XX. The linear increase of the $X$ line and the quadratic increase of the $\mathrm{XX}$ line at low excitation are evident. These correlated behaviours are the finger prints of the exciton/biexciton luminescences of an isolated quantum dot. The solid lines in figure $7 \mathrm{~b}$ represent the results of theoretical calculation obtained from the steady-state solutions of the rate equations based on the four-level exciton-biexciton level scheme (inset in

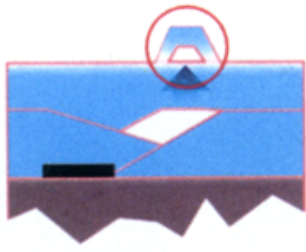

Upper InGaAs

+ Growth interruption
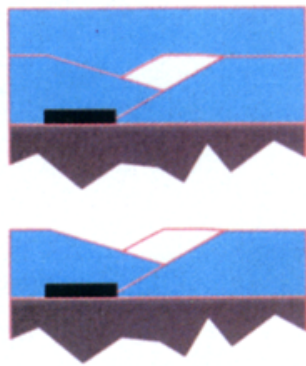

InGaAs

AlGaAs
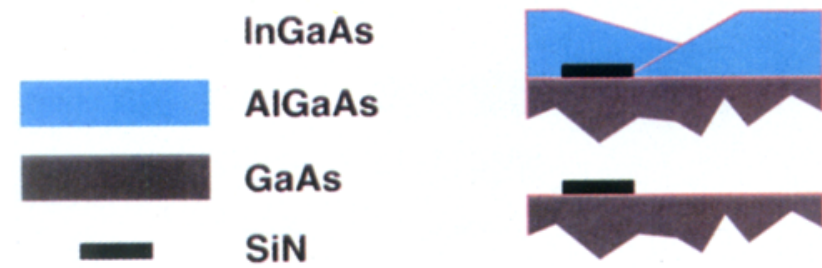

Bottom InGaAs + Growth interruption

1st AIGaAs

2nd AIGaAs

SiN patterning

Figure 9. Schematic representation of control of disk (nanocrystal) ordering by strain anchor. 
figure $7 \mathrm{~b}$ ) by assuming a ratio of the radiative lifetime of exciton relative to that of biexciton, $\tau^{\mathrm{x}} / \tau^{\mathrm{xx}}=2 \cdot 4$ (Kamada et al 1998). The agreement between the experimental results and the calculation is excellent. The exciton/biexciton pair attribution is confirmed. We conclude therefore that even in the quantum disk in mesoscopic confine regime the biexciton effect is substantial. The observed PL energy separation of $4.7 \mathrm{meV}$ between $\mathrm{X}$ and $\mathrm{XX}$ agrees well with the biexciton correlation energy, about $5 \mathrm{meV}$, expected for small $\mathrm{GaAs} / \mathrm{AlGaAs}$ quantum dot structures (Hu et al 1990). It is significantly enhanced compared to the value of about $1 \mathrm{meV}$ observed in QWs and that of about $0.2 \mathrm{meV}$ in bulk GaAs (Miller et al 1982; 't Hooft et al 1987).

\subsection{Excited state optical transitions of OD-exciton/biexciton system}

In this section, we shall discuss in detail optical excitation process, exciting exciton and biexciton of the present OD system. While carrying out photoluminescence excitation (PLE) measurement on an isolated dot luminescence, we observed multitude of excited state resonances all of which were equally sharp and distinct as the ground state emission. Figure 8 demonstrates such PLE spectra of the single dot exciton-biexciton pair taken at $3.8 \mathrm{~K}$; the quantum disk was exactly same as that in figure 7. Excitation light was linearly polarized. Under weak excitation (about $10^{2} \mathrm{~W} / \mathrm{cm}^{2}$ ), the PLE spectrum is characterized by a number of well-separated sharp resonances, which we attribute to the exciton excited states. In two PLE spectra, a number of sharp resonance features are common; nearly one-to-one correspondence is observed in the $\mathrm{X}$ and XX PLE peak features. Such one-to-one correspondence was also found by Brunner et al (1994). Here, the absorption of a photon creates an exciton of the angular momentum $I=1$. The subsequent photon absorption from the excited $I=1$ exciton state to the biexciton is allowed. At a low excitation density, the first photon absorption is dominant. For higher excitations at which the exciton state saturates, the second absorption prevails over the first process. The net absorption for the biexciton creation is proportional to the product of the energy-level density distribution of exciton and that of biexciton. Because the energy-level density distribution of the biexciton as product of two excitons, is much higher, the net absorption is determined by the sparse distribution of exciton levels. Accordingly, the biexciton PLE features resemble the exciton PLE features: it is the origin of the one-to-one correspondence between exciton and biexciton PLE features (Kamada et al 1998).

Having examined optical process in isolated single quantum dot, we have concluded that even in a mesoscopic dot, where the extent is more than twice the exciton Bohr radius, the exciton OD confinement leading to discrete density-of-states and substantially enhanced biexciton (or multi-exciton correlation), have significant roles.

\section{Control cites of self-organization}

Having examined physics of self-organization and optical property of the resulting quantum disks, we believe that

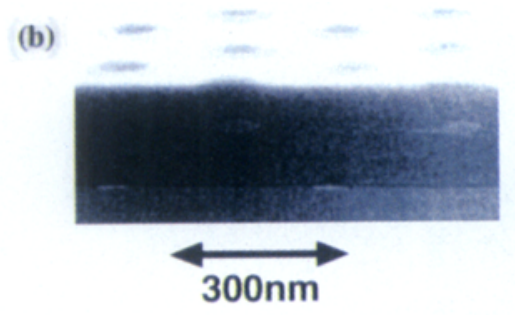

(a)
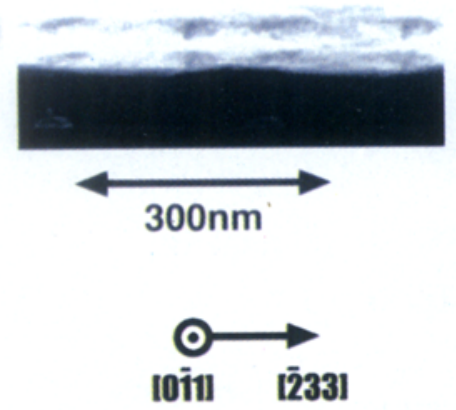

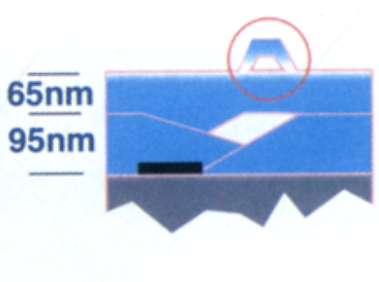

Upper InGaAs

+ Growth interruption

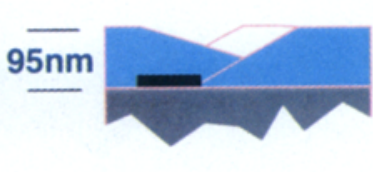

Bottom InGaAs

+ Growth interruption

InGaAs

AlGaAs

GaAs

SiN

Figure 10. SEM snap shots to nanocrystal ordering control, one after the first ordered InGaAs island formation and the other for the final structure (nominally $3.5 \mathrm{~nm}$ thick $\operatorname{In}_{0.25} \mathrm{Ga}_{0.75} \mathrm{As}$ ). For the details of the schematic representations of the SEM snap shot, refer the text of figure 9. 


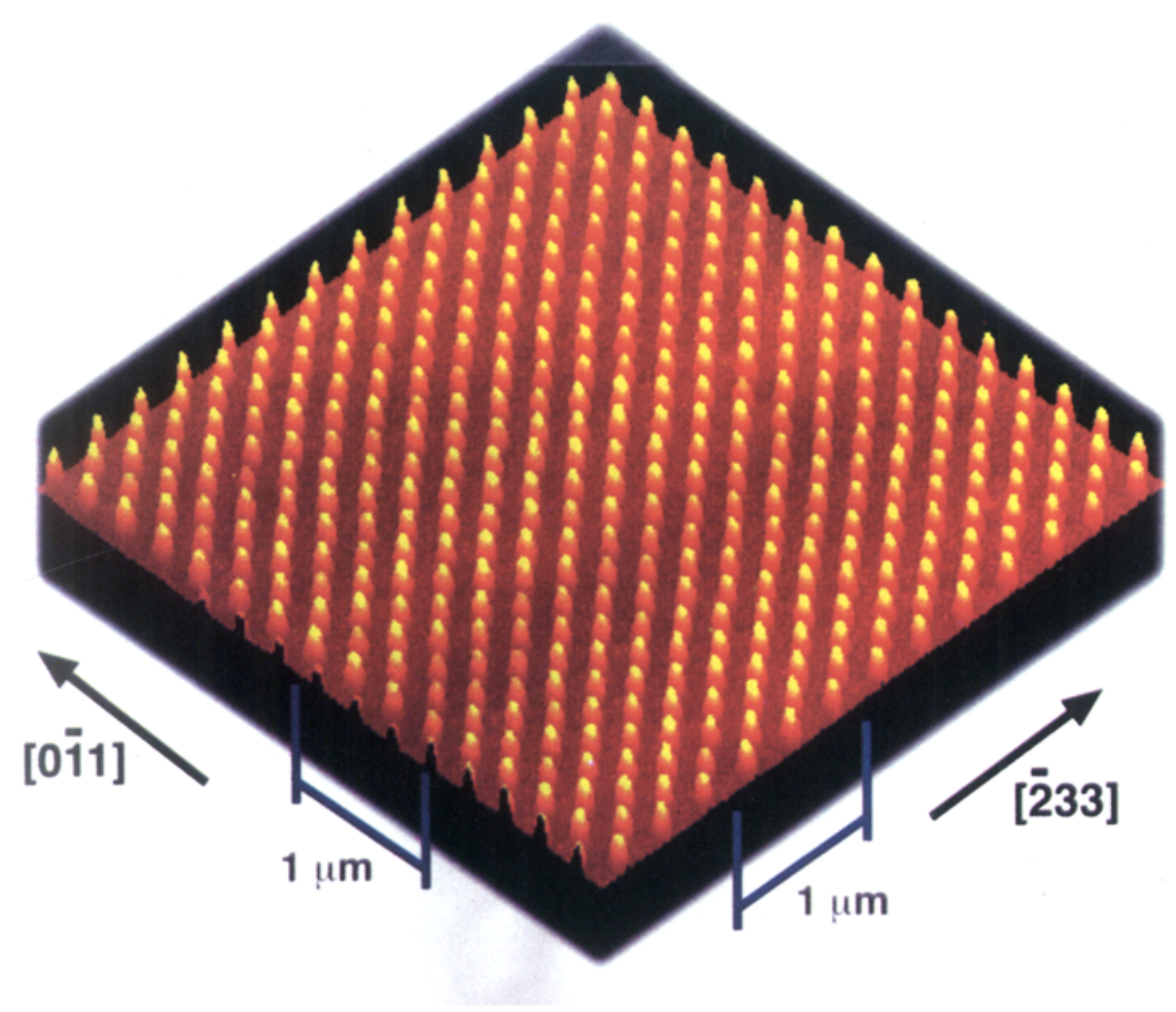

Figure 11. A example of large area ordering ( $3 \cdot 5 \mathrm{~nm}$ thick $\ln _{0.3} \mathrm{Ga}_{0.7} \mathrm{As}$ ).

these quantum disks serve as superior achievement of nano-fabrication assisted by nature and specifications of zero-dimensional quantum confinement. Remaining problem to be overcome is still the inhomogeneous statistical distribution of the structure and better ordering. These two seem essential for realistic device application whatever it aims at. We give results of our recent attempt to artificially position the self-organized structure more accurately by periodic seeding on the virgin (311)B substrate. Figure 9 is a schematic representation of control of disk (nanocrystal) ordering by strain anchor. Basic idea is simple: artificially ordered seeding may help the ordering of the self-aligned InGaAs strain anchors and thereby site-controlled self-organization proceeds just above strain anchor by vertical strain propagation (Kuramochi et al 1997). This was carried out in four steps: (i) We prepared patterned array of $\mathrm{SiN}$ islands on the virgin (311)B-oriented wafer. (ii) A growth of $\mathrm{AlGaAs}$ resulted in equally ordered array of pentagonal-shaped hollow due to facet development by competing faceorientation-dependent growth rate. (iii) Subsequent deposition of InGaAs leads to a preferential segregation into pentagonal hollow resulting in array of InGaAs islands which are equally well-ordered. (iv) This area selective growth of InGaAs islands was then used to control the sites of the self-organization by vertical strain propagation within a second InGaAs layer grown subsequently (Kuramochi et al 1997; 1998). These procedures produced a structure shown in figure 10, where two SEM images display snap shots, one after the first ordered InGaAs island formation and the other for the final structure (nominally $3.5 \mathrm{~nm}$ thick $\operatorname{In}_{0.3} \mathrm{Ga}_{0.75} \mathrm{As}$ ) (Kuramochi et al 1998). As the surface nanocrystals construct themselves by the supply of the material in neighbourhood, they serve as most beautiful ordering as array pitch is optimized, and a example of large area ordering (Kuramochi et al 1997) is given in figure 11. It presents excellent ordering of the nanocrystal, therefore it promises the future progress toward practical applications.

\section{Conclusion}

We have examined the spontaneous rearrangement of a strained InGaAs/AlGaAs heterostructure on a (311)Bsubstrate into naturally ordered array of InGaAs disks automatically buried in Al-rich alloy. We have stressed important interplay of islanding of the material with lattice mismatch, atomic diffusion across the interface between unstrained and strained materials, lateral mass transport, and development of the surface into low index surfaces with low surface energies. The damageless fabrication thus bestows the quantum disks with excellent optical properties. Single dot spectroscopy revealed that lateral together with vertical confinement of exciton motion not only discretizes the exciton density-of-states that result in sharp and distinct photo emission/absorption 
spectra but also enhances exciton-exciton correlation giving clear biexcitonic feature even within their mesoscopic confinement. This is an unique property of the zero-dimensional system.

Finally, we have proposed a novel periodic strain anchoring for the quantum disk ordering, excelling any results previously observed. Selective area growth of InGaAs islands, by using lithography-defined $\mathrm{SiN}$ dotmasks and subsequent lateral epitaxial overgrowth of AlGaAs on a (311)B face, enables preparation of beautifully ordered InGaAs island array, which was in turn used as strain anchors. Strain propagation to surface positions the final quantum disk arrays formed by self-organization process, which undergoes further self-organization by the supply of the material in neighbourhood, resulting in a precise ordering of the $\mathrm{SiN}$ dot masks.

\section{Acknowledgments}

We would like to thank R Nötzel, Paul Drude Institute, Prof T Fukui and Prof H Hasegawa, Hokkaido University, Professor S L Chuang, University of Illinois, and Drs T Takagahara, H Gotoh, A Chavez-Pirson, and M Notomi, NTT Laboratories for helpful discussions. We would like to thank Drs $\mathrm{N}$ Uesugi and $\mathrm{T}$ Makino, NTT Basic Research Laboratories for their continuous encouragement throughout this study.

\section{References}

Brunner K, Abstreiter G, Bohm G, Tränkle $G$ and Weimann $G$ 1994 Phys. Rev. Lett. 731138

Fafard S, Leon R, Leonard D, Merz J L and Petroff P M 1995 Phys. Rev. B52 5752

Forchel A, Steffen R, Koch T, Mitchel M, Albrecht M and Reiecke T L 1996 Semicond. Sci. Technol. 111529
Gammon D, Snow E S, Shanabrook B V, Katzer D S and Park D 1996 Phys. Rev. Lett. 763005

Grundmann M et al 1995 Phys. Rev. Lett. 744043

Hu Y Z et al 1990 Phys. Rev. Lett. 641805

Kamada H, Temmyo J, Notomi M, Furuta $\mathrm{T}$ and Tamamura $\mathrm{T}$ 1996 Extracted abstracts of 1996 int. conf. on solid state devices and materials (Business Centre for Academic Societies)

Kamada H, Temmyo J, Notomi M, Furuta T and Tamamura T 1997 Jpn J. Appl. Phys. 364194

Kamada H, Ando H, Temmyo J and Tamamura T 1998 Phys. Rev. $\mathbf{B 5 8} 16243$

Kuramochi E, Temmyo J, Tamamura T and Kamada H 1997 Appl. Phys. Lett. 71 1655, 3448

Kuramochi E, Temmyo J, Kamada H and Tamamura T 1998 Jpn J. Appl. Phys. 371559

Leonard D, Krishnamurthy M, Reaves C M, Denbaars S P and Petroff P M 1993 Appl. Phys. Lett. 633203

Leonard D, Krishnamurthy M, Fafard S, Merz J L and Petroff P M 1994 J. Vac. Sci. Technol. B12 1063

Marzin J-Y, Gerard J-M, Izrael A, Barrier D and Bastard G 1994 Phys. Rev. Lett. 73716

Miller R C, Kleinman D A, Gossard A C and Munteanu O 1982 Phys. Rev. B25 6545

Notomi M, Furuta T, Kamada $H$, Temmyo $J$ and Tamamura $T$ 1996 Phys. Rev. B53 15743

Nötzel R, Temmyo J and Tamamura T 1994a Nature 369 131

Nötzel R, Temmyo J and Tamamura T 1994b Jpn J. Appl. Phys. 33 L275

Nötzel R, Fukui T, Hasegawa $\mathrm{H}$, Temmyo $\mathrm{J}$ and Tamamura $\mathrm{T}$ 1995 Appl. Phys. Lett. 662525

Nötzel R, Temmyo J, Kamada $H$, Furuta $T$ and Tamamura $T$ 1996 Appl. Phys. Lett. 65457

Temmyo J and Tamamura T 1998 Jpn J. Appl. Phys. 37 1487

Temmyo J, Nötzel R and Tamamura T 1997 Appl. Phys. Lett. 711086

't Hooft G W, Poel W A J A v d, Molenkamp L W and Foxon C T 1987 Phys. Rev. B35 8281 